Boletín de la Sociedad Geológica Mexicana

XI Congreso Nacional de Paleontología

Volumen 61, nÚM. 2, 2009, P. iii-xii

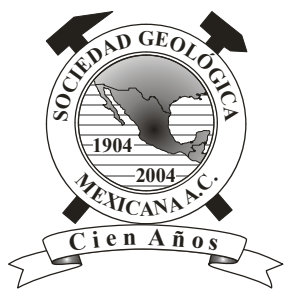

\title{
Editorial:
}

\section{Avances en el Boletín de la Sociedad Geológica Mexicana desde 2005 y perspectivas de futuro}

\section{Introducción}

A finales de 2005 entró en funciones un nuevo comité editorial del Boletín de la Sociedad Geológica Mexicana, que fue renovado parcialmente a finales de 2008. Ante el nuevo comité se encontraba el reto de continuar la publicación del Boletín como revista con periodicidad regular que siguiera constituyendo un punto de referencia para las Ciencias de la Tierra en México. El esfuerzo emprendido desde entonces vino precedido de la edición del excepcional Volumen del Centenario, que constituye el esfuerzo colectivo más notable hasta la fecha como contribución al Boletín y está llamado a convertirse en una obra de referencia por mucho tiempo. Tal precedente constituyó la base pricipal, en 2007, de la inclusión del Boletín en el Índice de Revistas Mexicanas de Investigación Científica y Tecnológica de Conacyt. Sin embargo, el reto real para el Boletín tras el Volumen del Centenario no lo constituye superar a éste en ningún aspecto, sino garantizar la periodicidad del Boletín y aumentar la calidad y cantidad de artículos publicados en éste al mismo tiempo, en base a un esfuerzo sostenido.

El principal problema que afrontó el Boletín en 2005 fue el mismo que ya enfrentaron los comités editoriales anteriores: la escasez de artículos sometidos de forma espontánea para su publicación, toda vez que los autores han dirigido sus esfuerzos en publicar preferencialmente sus trabajos en revistas pertenecientes al Science Citation Index. Así, durante años (incluyendo el mismo Volumen del Centenario), las contribuciones al Boletín se produjeron mayoritariamente por invitación. Mediante tal mecanismo, resulta obvio que el futuro de cualquier revista es dependiente casi en exclusiva de la persistencia de sus responsables, e independiente de su línea o de su alcance editorial como publicación regular. Por lo tanto, esa situación constituía un factor de riesgo para el Boletín que tenía que recibir la debida atención.

No es ninguna sorpresa que la inclusión en el Índice de Conacyt haya conllevado un aumento considerable en el sometimiento espontáneo de artículos al Boletín. Al fin y al cabo, a pesar de que el Boletín ha contado desde hace tiempo con el debido arbitraje, para los académicos publicar en una revista incluída en el Índice de Conacyt es curricularmente mucho más interesante que publicar en una revista que no lo esté. Para conseguir tal indización, la edición del Volumen del Centenario durante el ejercicio del comité editorial anterior ha sido fundamental, con lo que es indudable que los logros del comité editorial actual están fundados en los logros del comité editorial anterior. Sin embargo, sin un esfuerzo sostenido que garantizara aspectos como la debida periodicidad, la calidad de las contribuciones y de su arbitraje, la presencia internacional del Boletín, etc., el Volumen del Centenario tal vez no hubiera contribuído a la continuidad de esta revista.

A continuación, se presenta una relación de las acciones que se han tomado en el Boletín desde finales de 2005, su situación actual, y diversos aspectos relevantes en cuanto a las contribuciones publicadas en los últimos años.

\section{Cambios en el Comité Editorial}

Al decidirse el cese en sus funciones del comité editorial en ejercicio hasta finales de 2005, fue preciso definir la composición renovada del comité entrante en el que entró una gran mayoría de nuevos miembros, aunque permanecieron varios del comité saliente. La experiencia de éstos ha probado ser fundamental para sostener de forma adecuada las funciones del nuevo comité. Una de las características principales del comité saliente era la escasa diversidad institucional que representaban sus miembros, con una gran preeminencia de académicos pertenecientes a las entidades de edición del Boletín, a saber, el Instituto de Geología o el Centro de Geociencias de la UNAM (en diferentes épocas). Con el nuevo comité editorial, con mayor cantidad de miembros, se aumentó la diversidad institucional de éstos, incorporando inicialmente académicos de varias universidades mexicanas como la Michoacana de San Nicolás de Hidalgo, la Autónoma de Nuevo León, la Autónoma de San Luis Potosí o la Autónoma de Baja California Sur y, posteriormente, del CICIMAR del Instituto Politécnico Nacional. Asimismo, se incorporaron varios editores procedentes de instituciones extranjeras (universidades de Arizona, Barcelona y São Paulo). La Universidad Nacional Autónoma de México, por su parte, aportó la representación más 
nutrida, con académicos de los institutos de Geología y Geofísica, el Centro de Geociencias y la Facultad de Ingeniería, totalizando 13 de los 22 miembros del comité editorial. Asimismo, durante la selección de los nuevos miembros del comité se procuró también contar con la máxima pluralidad posible en cuanto a las disciplinas de las Ciencias de la Tierra representadas por éstos.

A finales de 2008 se produjo la renovación parcial del comité editorial a fin de continuar incidiendo en los objetivos y funciones que se determinaron para éste a partir de 2006. En la misma línea de la configuración del comité en 2005 , se incrementó la pluralidad tanto de las instituciones mexicanas como de las extranjeras, y se redujo la representación de la UNAM. En la nueva configuración están representados el Instituto Politécnico Nacional, el Instituto Mexicano del Petróleo, y las universidades autónomas de Guerrero, San Luis Potosí y Nuevo León, e instituciones extranjeras como el Instituto Geológico de Catalunya, el Museo de Fósiles de Mizunami (Japón), y las universidades de Barcelona, Ginebra, del Estado de Nuevo México, de Texas en El Paso, Nacional de La Plata, y Nacional de Colombia (totalizando una representación de seis países distintos, además de México). La representación de la UNAM, por su parte, se redujo a 9 de 22 miembros aunque aumentó la pluralidad institucional de los mismos al incorporarse un académico de la Facultad de Ciencias. Todo ello, sin reducir la multidisciplinariedad del colectivo.

\section{Un Boletín electrónico de acceso libre}

Con el objetivo de garantizar la máxima difusión de los trabajos publicados en el Boletín y, por lo tanto, para maximizar la diseminación del conocimiento contemplada por la Sociedad Geológica Mexicana desde sus inicios, todo el contenido del Boletín desde 1904 se colocó en la red mundial desde la gestión del comité editorial anterior. Desde entonces, el contenido completo del Boletín es de acceso libre e irrestricto. Asimismo, con la entrada en funciones del nuevo comité editorial, una de las primeras decisiones que se tomaron fue la de publicar el Boletín en lo sucesivo exclusivamente de forma electrónica. Sin embargo, se mantiene abierta la posibilidad de editar en formato convencional alguno de los números ya publicados electrónicamente, en función del interés de los lectores o de los mismos autores que contribuyeron con sus trabajos, $\mathrm{y}$ de la disponibilidad de fondos para costear la publicación en papel.

\section{Inclusión en el Î́ndice de Conacyt}

Además de resultar un factor decisivo para garantizar el sometimiento espontáneo de artículos al Boletín, su inclusión en el Índice de Revistas Mexicanas de Investigación Científica y Tecnológica de Conacyt garantiza una cierta autonomía presupuestal de forma estable. Ésta es importante al acometer la edición técnica de los artículos, pues se trata de una actividad remunerada para las personas involucradas en ella que no poseen un cargo académico.
Sin embargo, la entrada del Boletín en el Índice de Conacyt tiene una vigencia de dos años, con el siguiente condicionamiento: "deberá ampliar el número de árbitros, incluyendo investigadores de otras instituciones nacionales, preferentemente que pertenezcan al SNI y del extranjero. Así mismo, incrementar el número de artículos de otras instituciones y del extranjero, solicitar su ingreso en indices y resúmenes internacionales". La próxima evaluación que recibirá el Boletín por parte de Conacyt está prevista durante el presente año 2009.

En algunas de las secciones a continuación se explica de qué manera se han seguido todas las indicaciones emitidas por el comité de evaluación de Conacyt.

\section{Incidiendo en diversas tipologías de trabajos publicables}

Durante las funciones de los dos comités editoriales anteriores, los trabajos publicados en el Boletín se caracterizaron por ser mayoritariamente artículos de investigación y revisiones temáticas. Sin embargo, también pueden tener cabida artículos de difusión o divulgación, notas cortas, rápidas o de carácter técnico, reseñas, bases de datos $\mathrm{u}$ otros tipos de trabajos. El contenido deseable de los trabajos que pueden publicarse en el Boletín, a diferencia de las revistas mexicanas que están incluídas en el Science Citation Index, abarca trabajos de ámbito local, trabajos técnicos o basados en la implementación o aplicación de una técnica, y artículos de investigación aplicada. Ello, tanto a nivel de trabajos de investigación originales como a nivel de revisiones remáticas. Hasta la fecha, existe una coordinación efectiva y permanente con el comité editorial de la Revista Mexicana de Ciencias Geológicas que permite a ambas revistas publicar artículos de tipo y ámbito distinto, en el sentido expuesto arriba.

Por otro lado, el Boletín puede constituir el medio ideal para la publicación de datos obtenidos en tesis de maestría, o inclusive en tesis de licenciatura, que muchas veces permanecen inéditos, pues los distintos programas de posgrado relacionados con Ciencias de la Tierra en México plantean la exigencia de publicación sólo a nivel de doctorado. Así, el Boletín puede ser un medio útil de iniciación en la publicación de artículos científicos para muchos estudiantes.

El objetivo de todo ello no es sólo dotar al Boletín de un contenido propio y diferenciado de otras revistas mexicanas, sino publicar trabajos cuyo contenido típicamente se considera como "insuficiente" en revistas internacionales por tratarse de trabajos en los ámbitos local o técnico. Naturalmente, tal insuficiencia no se refiere a la calidad o representatividad de los datos expuestos sino al alcance geográfico o geológico de los mismos. De forma análoga, la aplicación de una sóla técnica o metodología analítica (o de unas pocas) puede llevar a la obtención de datos científica o regionalmente relevantes, aunque el alcance del trabajo no alcance el perfil deseable de ciertas revistas. Trabajos de este tipo no tienen en la actualidad la difusión adecuada en 


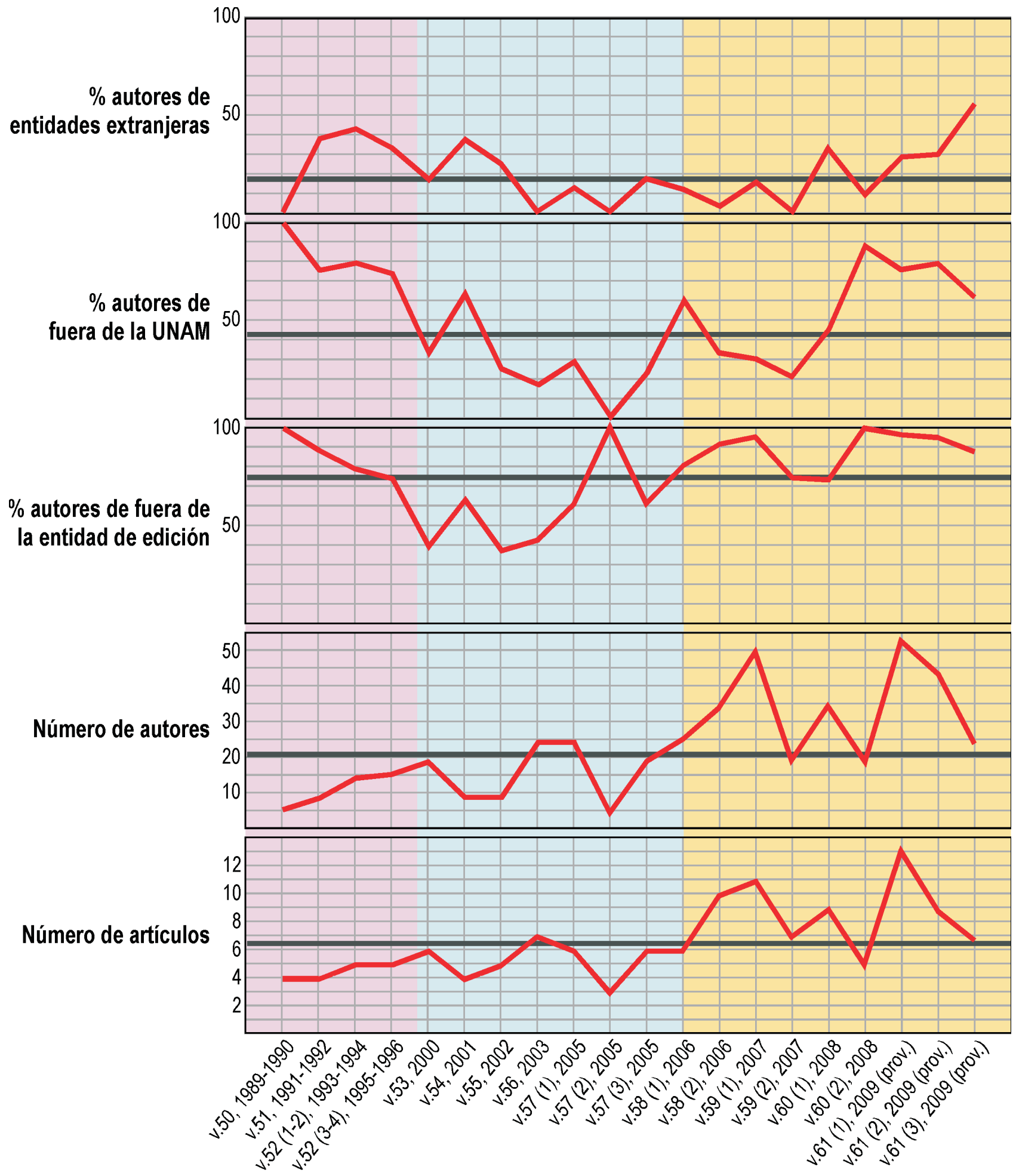

Figura 1. Evolución de diversas variables en la cantidad de autores y de artículos publicados en el Boletín de la Sociedad Geológica Mexicana desde 1989 hasta Febrero de 2009, por números editados. Las franjas de color en vertical representan el alcance de los tres diferentes comités editoriales en ejercicio durante el periodo, y las líneas gruesas horizontales representan el valor promedio de cada variable sin contar con los valores del vol. 61, cuya composición es de carácter provisional en el momento de elaboración de la figura 
las revistas científicas de Ciencias de la Tierra, por lo que en el Boletín se desea hacer de ello una razón de ser.

\section{Diversidad temática de los artículos publicados}

El ámbito temático del Boletín son todas las Ciencias de la Tierra y todos aquellos temas relacionados con las mismas. Sin embargo, la variedad temática de los trabajos publicados en el Boletín no reflejaba debidamente las líneas de trabajo existentes en las instituciones mexicanas dedicadas al estudio de las Ciencias de la Tierra. Ejemplos de ello son la relativa escasez de trabajos sobre paleontología, geomorfología o geoquímica isotópica durante las dos últimas décadas, o la histórica ausencia casi completa de trabajos de geología marina. Además del enfoque tradicional a partir de disciplinas o subdisciplinas de las Ciencias de la Tierra tales como la geología estructural, la estratigrafía o la mineralogía, no hay que desdeñar que en la actualidad muchas de éstas muestran un enfoque multidisciplinario o transdisciplinario. Tal es el caso de la geología urbana, la geoarqueología, la mineralogía médica, la geología ambiental o la geoquímica de fluidos. Es decir, sin olvidar las disciplinas tradicionales de las Ciencias de la Tierra o las que tradicionalmente han contribuido más extensamente al Boletín, es deseable que éste sea un reflejo lo más fiel posible de la labor actual en las Ciencias de la Tierra. Por ello, los números especiales que se han editado desde 2006 han sido tres de geología urbana y uno de geología marina. Asimismo, están abiertas las convocatorias de sendos números especiales dedicados a paleoclimatología, a paleontología, y a aplicaciones de teledetección y sistemas de información geográfica. Asimismo, se está explorando la posibilidad de editar en un futuro números especiales de geología ambiental y de sedimentología.

Fruto de dicha franca intencionalidad y de la misma composición plural del comité editorial del Boletín, entre el vol. 58 no. 2 de 2006 y el vol. 60 no. 2 de 2008, se han publicado 41 trabajos pertenecientes a más de 15 disciplinas y subdisciplinas diferentes en Ciencias de la Tierra (Tabla 1). Entre ellos, dominan los temas de geomorfología como consecuencia de la edición de los tres números de geología urbana (que incluyen 16 artículos, 4 notas técnicas y 2 reseñas), y es notable la presencia de trabajos relacionados con la sismología, la estratigrafía, la mineralogía y los yacimientos minerales. Asimismo, el número especial de geología y ambientes deposicionales marinos (vol. 61 no. 1 de 2009) incluirá trabajos de sedimentología, geoquímica elemental, geoquímica orgánica, biogeoquímica, geofísica marina, limnología, hidrografia e ingeniería.

\section{Cantidad de contribuciones}

La cantidad de trabajos publicados en el Boletín ha aumentado considerablemente desde la entrada en funciones del actual comité editorial (Figura 1 y Tabla 2), de forma que se prevé que la cantidad de artículos publicados entre 2006 y 2009 (4 años) sea más del doble que los publicados entre 2000 y 2005 ( 6 años) y cinco veces más que los publicados
Tabla 1. Trabajos publicados en el Boletín de la Sociedad Geológica Mexicana entre el vol. 58 no. 2 de 2006 y el vol. 60 no. 2 de 2008 (ambos inclusive), por disciplinas o subdisciplinas.

\begin{tabular}{lc}
\hline Disciplina o subdisciplina & $\begin{array}{c}\text { Número de } \\
\text { contribuciones }\end{array}$ \\
\hline Geomorfología & 8 \\
Sismología & 4 \\
Mineralogía & 4 \\
Estratigrafía & 4 \\
Yacimientos minerales & 3 \\
Hidrogeología & 2 \\
Geología estructural & 2 \\
Paleontología & 2 \\
Riesgo geológico/geotecnia & 2 \\
Geoquímica de fluidos & 2 \\
Reseñas de libros & 2 \\
Volcanología & 1 \\
Edafología & 1 \\
Geoarqueología & 1 \\
Geología ambiental & 1 \\
Modelado analógico & 1 \\
Otros temas & 1 \\
Total de contribuciones: & 41 \\
\hline
\end{tabular}

entre 1989 y 1996 (8 años). La cantidad de páginas publicadas (Figura 1 y Tabla 3 ) presenta un comportamiento relativo similar al de la cantidad de trabajos aunque, en cantidades absolutas, la producción del periodo 20002005 y del periodo 20062009 es muy similar. Ello es debido a la "distorsión" que supone la existencia del Volumen del Centenario ( 3 números en 2005 y 1 en 2006), que contuvo exclusivamente extensas revisiones temáticas.

Sin embargo, para evaluar correctamente el sometimiento de artículos al Boletín es preciso considerar no sólo los artículos publicados sino también los rechazados. Como se aprecia en la Figura 2, la cantidad total de artículos sometidos al Boletín ha ido en aumento desde 2004, alcanzando un máximo de 66 artículos sometidos en 2008, el año en que se dió a conocer la inclusión del Boletín en el Î́ndice de Conacyt. Asimismo, aumentó en mayor medida la cantidad de artículos sometidos al Boletín de forma espontánea. Por tanto, se puede interpretar que tanto el aumento en la cantidad de trabajos enviados al Boletín como el aumento en el carácter espontáneo de los mismos son consecuencia directa de la inclusión del Boletín en el Índice de Conacyt.

\section{Diversidad institucional de los autores de artículos}

La diversidad institucional de los autores que han contribuido al Boletín ha sido tradicionalmente elevada (ver datos hasta 1996 en la Figura 1). Igualmente, el Boletín 
Tabla 2. Cantidad de trabajos publicados por año en el Boletín de la Sociedad Geológica Mexicana desde 1989 hasta la actualidad. Las franjas de color horizontales indican los diversos comités editoriales en ejercicio. Los datos de los números correspondientes a 2009 no son definitivos. La línea roja indica las variaciones en periodicidad a través del tiempo.

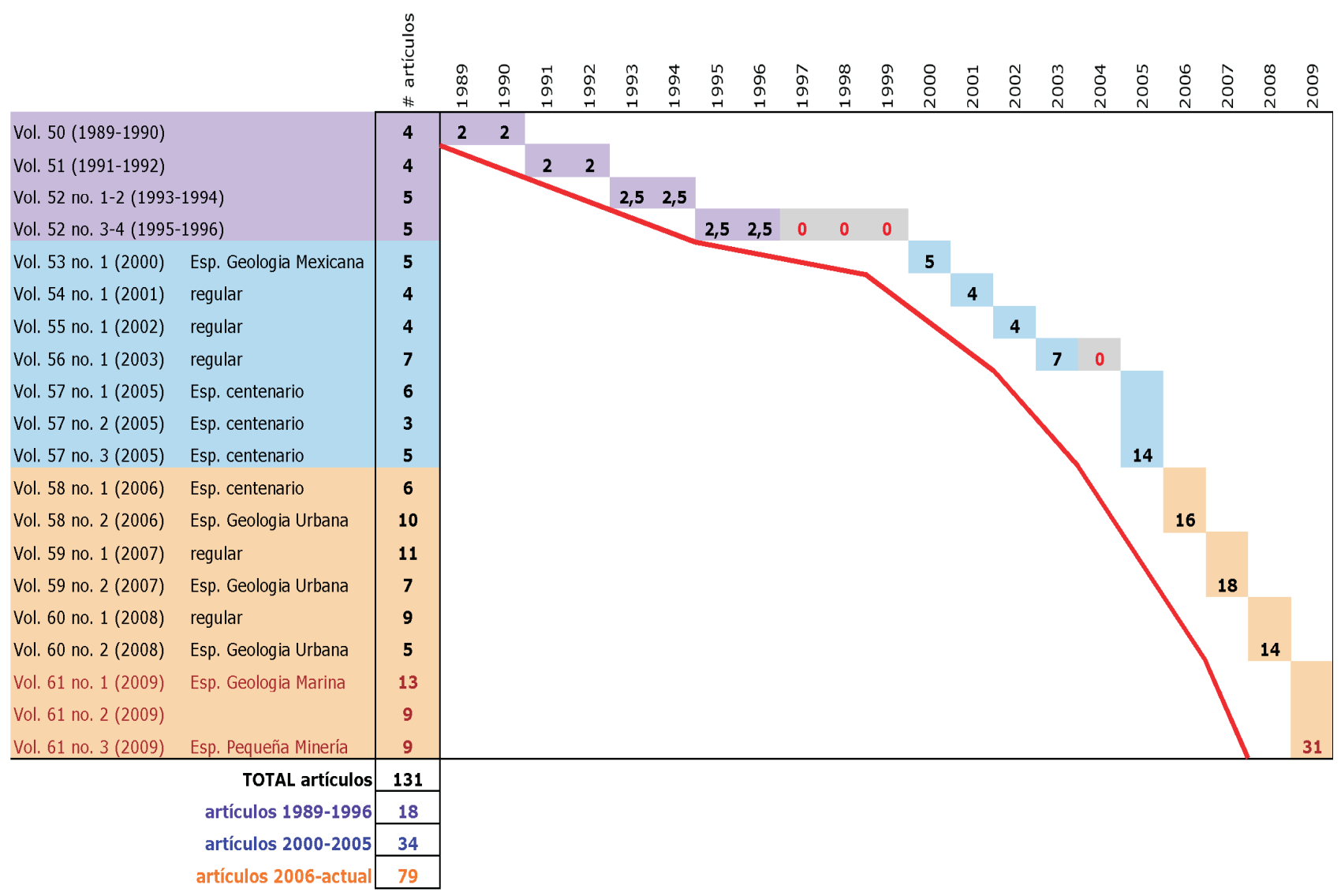

ha publicado históricamente gran cantidad de trabajos de autores procedentes de instituciones extranjeras, tanto en español como en inglés. Sin embargo, durante la última década, las contribuciones por parte de autores de instituciones pertenecientes a la UNAM se convirtieron en mayoritarias. La principal causa de esta especie de "monopolización" por parte de autores de la UNAM es posiblemente que esta universidad contiene la mayoría de las instituciones académicas en Ciencias de la Tierra con mayor población académica en México, que son los institutos de Geología, Geofísica y Geografía, el Centro de Geociencias, y las facultades de Ingeniería y de Ciencias, además del Instituto de Ciencias del Mar y Limnología, el Centro de Ciencias Atmosféricas y otras dependencias cuya investigación está relacionada con las Ciencias de la Tierra.

Este problema fue detectado desde el inicio del ejercicio del comité editorial actual y fue igualmente señalado por el comité de evaluación del Índice de Conacyt, por lo que se tomaron medidas encaminadas a fomentar el sometimiento de trabajos por parte de autores del extranjero y de otras entidades académicas mexicanas. En ese sentido es que la primera medida fue conformar un comité editorial plural, con presencia de académicos del extranjero, que aumentó al renovarse parcialmente dicho comité a finales de 2008.
El aumento en la cantidad de autores y de artículos por número editado vino acompañado por un aumento en la cantidad de autores adscritos a instituciones extranjeras, tanto a nivel absoluto como relativo (Figuras 1 y 3 ), y de un considerable aumento en la cantidad de contribuciones de instituciones mexicanas fuera de la UNAM. Así, durante el bienio 2005-2006, de los 152 autores que sometieron trabajos al Boletín, 86 pertenecían a la UNAM (56.6\%), 49 a otras instituciones mexicanas (32.2\%), y 17 a instituciones de otros 5 países (11.2\%). En cambio, durante el bienio 2007-2008, de los 296 autores que sometieron sus trabajos al Boletín, 88 pertenecen a la UNAM (29.7\%), 104 a otras instituciones mexicanas (35.1\%), y 104 a instituciones de otros 17 países (35.1\%). Ver detalle en la Tabla 4. Es decir que, de un bienio a otro, la cantidad de autores de la UNAM que sometieron trabajos al Boletín se mantuvo constante, mientras que la cantidad de autores de otras instituciones mexicanas se duplicó y la cantidad de autores de instituciones extranjeras se sextuplicó, para un total de autores de casi el doble. La lectura que se puede extraer de ello es difícilmente equívoca: el aumento en la cantidad de autores y de trabajos durante el último bienio es debida principalmente a autores de fuera de la UNAM, tanto de otras instituciones mexicanas como del extranjero. 
Tabla 3. Cantidad de páginas publicadas por año en el Boletín de la Sociedad Geológica Mexicana desde 1989 hasta la actualidad. Las franjas de color horizontales indican los diversos comités editoriales en ejercicio. Los datos de los números correspondientes a 2009 no son definitivos.

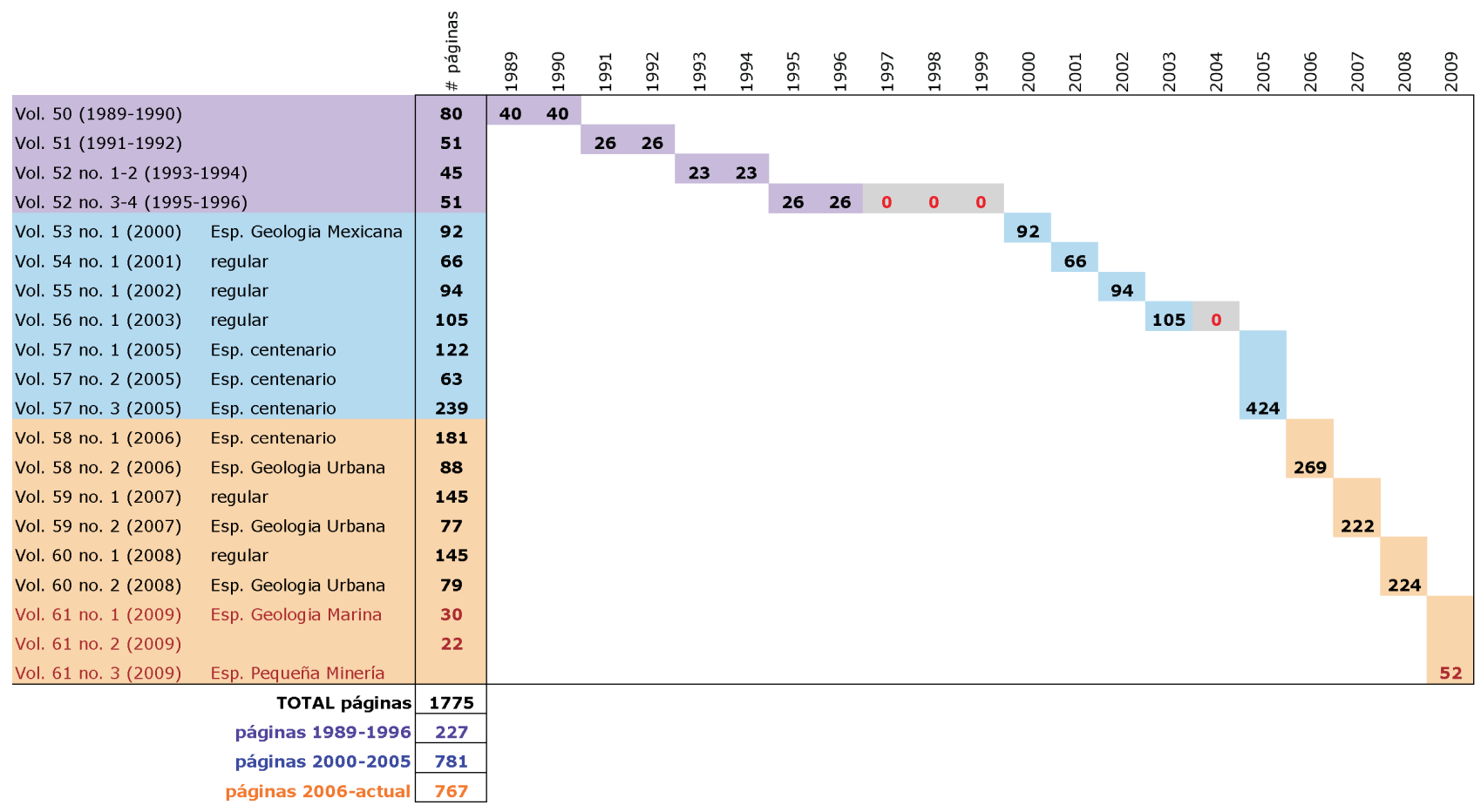

Es claro que la pertenencia del Boletín al Índice de Conacyt constituye un reclamo importante para los académicos y estudiantes de instituciones mexicanas, pero ello normalmente no constituye un factor a tener un cuenta para los autores de instituciones de otros países. Naturalmente, si estos autores buscan publicar en revistas del Science Citation Index el Boletín difícilmente les resultará una solución atractiva para la publicación de sus trabajos. Sin embargo, para éstos, el Boletín puede resultarles útil y amigable al garantizárseles un proceso de edición académica rápido y efectivo, la rápida publicación y libre disponibilidad en red de todos los trabajos, la falta de necesidad de limitar la extensión de los mismos, o la posibilidad de incluir figuras a color sin restricción. Además, la edición de números temáticos puede elevar el interés de los autores en publicar en el Boletín.

\section{Diversidad institucional del padrón de revisores}

Durante el bienio 2005-2006 se emitieron 53 revisiones para el Boletín, de las que 46 pertenecieron a académicos de 7 dependencias la UNAM (86.8\%), 4 de otras 4 instituciones mexicanas (7.5\%), y 3 procedentes de 2 instituciones de otros dos países $(5.7 \%)$. En cambio, durante el bienio 2007-2008 (Tabla 5) se emitieron 175 revisiones, de las que 47 pertenecieron a académicos de 13 dependencias la UNAM (26.9\%), 38 de otras 20 instituciones mexicanas (21.7\%), y 90 procedentes de 65 instituciones de otros 18 países $(51.4 \%)$. Entre el personal de instituciones mexicanas, el $83.5 \%$ de ellos pertenecen al Sistema Nacional de Investigadores. Así, la cantidad de revisiones emitidas desde la UNAM se mantuvo constante pero se duplicó la cantidad de dependencias de esta universidad que participaron en el proceso editorial. Ello es debido a que durante el bienio 2007-2008 se estableció y se mantuvo intencionalmente un equilibrio en el padrón de revisores teniendo en cuenta su entidad de adscripción. Ejemplo de ello es que las instituciones que más contribuyeron con revisiones durante el bienio 2005-2006 fueron el Centro de Geociencias y el Instituto de Geología con 21 y 10 revisiones, respectivamente (casi el $60 \%$ del total de revisiones), mientras que durante elbienio 2007-2008 contribuyeron con 10 y 11 revisiones. Ello, a pesar de que la cantidad de revisiones entre un bienio y el anterior es $31 / 2$ veces mayor. De esta forma, se ha cancelado el peso excesivo en las revisiones de una institución o de una dependencia de ésta en concreto. Ello, sin que haya disminuido la calidad de las revisiones o la categoría académica de los revisores.

El padrón de revisores durante el bienio 2007-2008 incluye, entre otros, académicos de instituciones como las universidades de Hamburgo, Kiel, Bremen y Giessen, el GFZ de Potsdam (Alemania), las universidades nacionales de La Plata y del Litoral, el CAICCONICET (Argentina), la Universidad de Leoben (Austria), las universidades de Ceará y Rio Grande do Sul (Brasil), la Universidad de Calgary (Canadá), las universidades de Chile y Austral de Chile (Chile), la Universidad Nacional de Colombia (Colombia), la Universidad de Pinar del Río (Cuba), las universidades de Barcelona, Granada, Oviedo, Complutense de Madrid, Politècnica de Catalunya, el CSIC (España), las 
Tabla 4. Autores por país y por número editado en el Boletín de la Sociedad Geológica Mexicana desde 1989 hasta la actualidad. Las franjas de color horizontales indican los diversos comités editoriales en ejercicio. Los datos de los números correspondientes a 2009 no son definitivos.

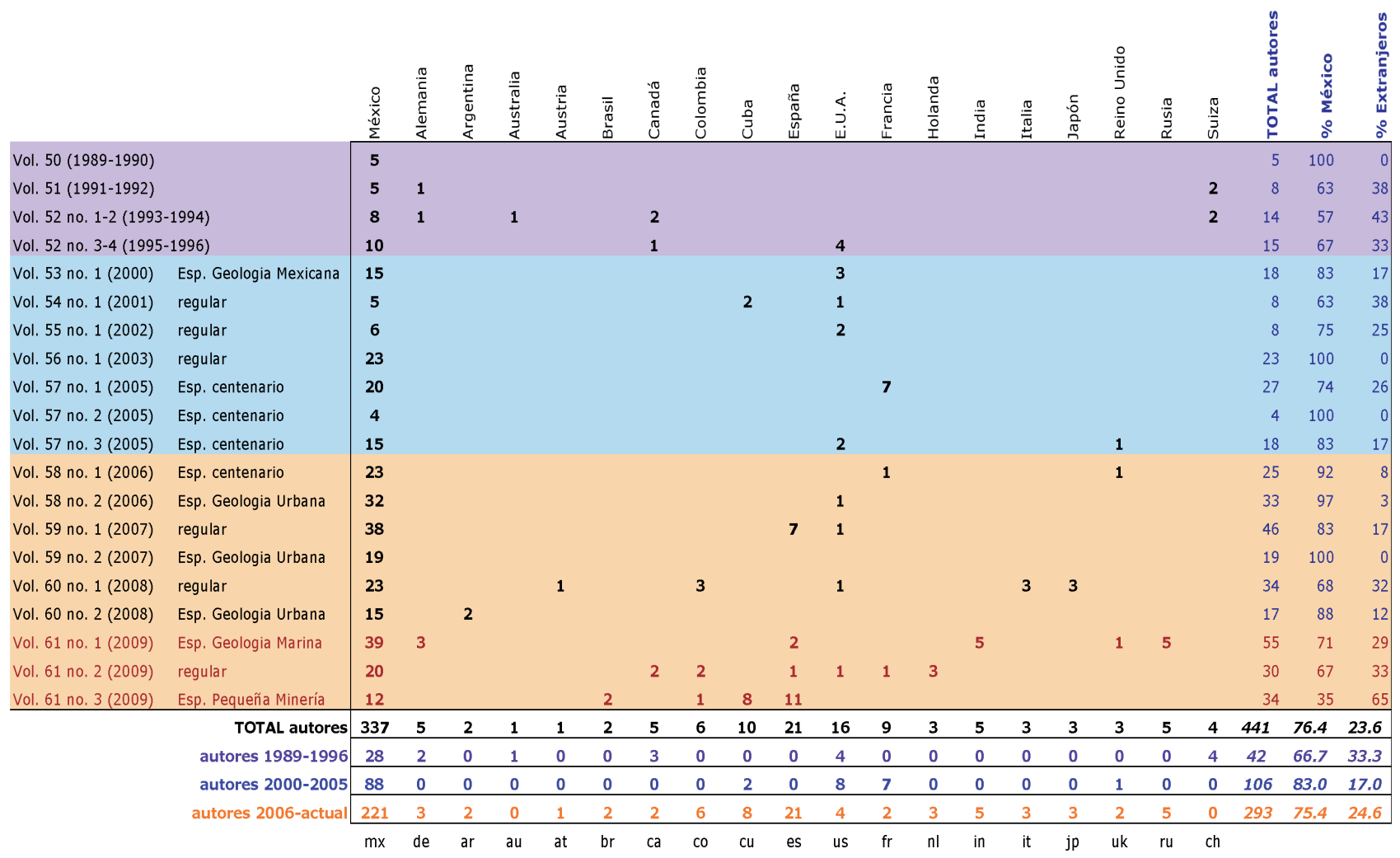

universidades de Arizona, California (San Diego), Minnesota, Colorado (Boulder), Texas (Austin y El Paso), Nueva York, estatal de Kent, estatal de Nuevo México, estatal de California, el USGS (Estados Unidos), la Universidad de Rennes y el CNRS (Francia), el Oertijdmuseum De Groene Poort (Holanda), la Universidad de Módena y el Museo de Historia Natural de Milán (Italia), el Museo de Fósiles de Mizunami (Japón), la Universidad de Kingston y el Laboratorio Marino de Plymouth (Reino Unido), las universidades de Ginebra, Basilea y Berna, la ETH de Zürich (Suiza), la Universidad de Chulalongkorn (Tailandia), o la Universidad Central de Venezuela (Venezuela).

\section{Arbitraje estricto e índice de rechazo}

No todos los trabajos son aceptados para revisión, pues algunos son rechazados directamente por uno de los editores, generalmente el editor en jefe. Ahora bien, los trabajos que pasan a revisión lo hacen bajo la supervisión de un editor, quien encarga como mínimo dos revisiones a especialistas en el tema de trabajo. Desde 2006 hasta Febrero de 2009 se han sometido al Boletín 116 artículos de los que han sido rechazados 36 (Figura 2), lo que corresponde al $31 \%$. Entre 2004 y 2005, en cambio, durante la recepción de trabajos para el Volumen del Centenario, se recibieron 26 trabajos, de los que se rechazaron 4 (15.4\%). Es decir, el índice de rechazo se ha duplicado durante el ejercicio del presente comité editorial, respecto a los datos disponibles del ejercicio del comité anterior. En sentido absoluto, sin embargo, ello representa que la cantidad de rechazos se ha octuplicado.

A pesar de los rechazos, se tiene especial cuidado en que los dictámenes sean siempre lo más propositivos posible. Fruto de ello, algunos manuscritos inicialmente rechazados han sido resometidos $\mathrm{y}$, con un nuevo arbitraje, han sido finalmente aceptados para su publicación.

\section{Periodicidad}

El comité editorial actual asumió sus labores con el encargo de mantener, cuanto menos, una periodicidad semestral (2 números al año), que es uno de los requisitos mínimos para poder optar a acceder al Índice de Conacyt. Aunque se partía de la edición exitosa del volumen del Centenario (con tres números en 2005 y uno en 2006), en las últimas décadas la periodicidad del Boletín había sido bianual entre 1989 y 1996, anual entre 2000 y 2003, mientras que el Boletín no se publicó entre 1997 y 1999, y en 2004 (Tablas 2 y 3). Entre los números 2 de 2006 y de 2008 se ha mantenido una periodicidad semestral en base a la alternancia de números regulares y números especiales. Para 2009 se prevé iniciar con una periodicidad cuatrimestral, con tres números por año, incluyendo dos números especiales y uno regular. La alternancia entre números regulares y especiales, aunque es un mecanismo que tiene sus desventajas, tiene la ventaja de poder agrupar temáticamente algunos artículos, de 
garantizar la periodicidad con números que contengan una cantidad significativa de artículos, y de atraer a algunos autores para publicar sus trabajos en números temáticos.

\section{Rapidez de la edición científica y puntualidad}

En promedio, el tiempo transcurrido entre la recepción de un artículo y su dictamen de aceptación o rechazo, incluyendo el tiempo de revisión y de corrección de los trabajos, ha disminuído de forma sostenida desde los 9.3 meses en 2005, 5.7 meses en 2006, 3.4 meses en 2007, hasta 2.7 meses en 2008 (aunque los datos de este año no son definitivos). Es decir, el proceso de edición científica se ha hecho más eficiente año con año, a pesar del notable aumento en la cantidad de trabajos sometidos al Boletín.

No obstante, la eficientización en la edición científica no es siempre garante de que los trabajos estén editados a tiempo para las fechas en que deben de estar terminados los números de la revista (Junio y Diciembre en periodicidad semestral; Abril, Agosto y Diciembre en periodicidad cuatrimestral). Ello es debido a que los cambios que se están produciendo en la edición técnica han provocado, en algunos casos, serios retrasos en la edición final.

\section{Visibilidad y distribución en la red mundial}

En 2007 se estableció una nuevo sitio para el Boletín en la red mundial (http://boletinsgm.igeolcu.unam.mx) que, a diferencia del sitio anterior, está administrado por medio de un servidor propio para los contenidos del Boletín, ubicado en el Instituto de Geología de la UNAM y proporcionado por dicha institución en apoyo al mismo. Este sitio incluye un despliegue más amigable de los diferentes números del Boletín, una sección para las listas de los 50 artículos más descargados (elaboradas por trimestres y años), así como algunas innovaciones recientes como el índice completo por autores, y cumple con las especificaciones de Conacyt. También comprende la posibilidad de establecer foros de opinión y una sección de noticias de interés para los lectores del Boletín. Todos los boletines desde 1904 están disponibles en dicho sitio.

Asimismo, al sitio en la red se le agregó una herramienta de generación de todo tipo de estadísticas de acceso, que permite estimar la difusión mundial del Boletín (Tabla 6). Para el año 2008, la mayoría de accesos al Boletín, además de México, se registraron desde Sudamérica, Europa, Estados Unidos, Centroamérica y el Caribe, y Japón. En total, hubo accesos al Boletín desde casi 90 países diferentes.

La visibilidad internacional del Boletín también aumentó a partir de su inclusión en Geoscience eJournals, un anillo en la red que contiene 51 revistas en Ciencias de la Tierra de acceso libre. En fechas recientes también se firmó un convenio con EBSCO para integrar al Boletín en el EBSCOhost Electronic Journals Service (EJS), un servicio que contiene miles de revistas electrónicas de todo tipo. Más recientemente, el Boletín fue incorporado al catálogo de

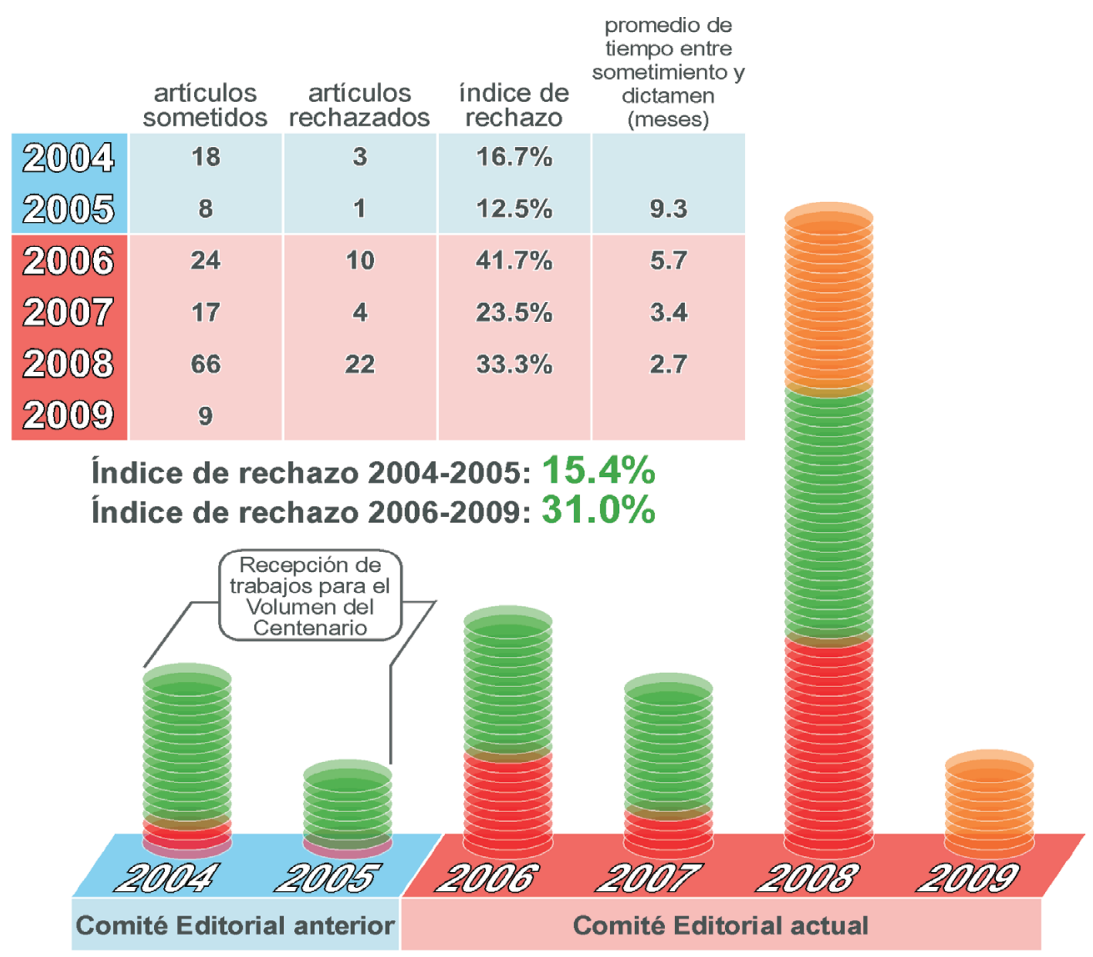

Figura 2. Representación diagramática mostrando la cantidad de artículos rechazados (en rojo), aceptados (en verde) y en proceso de revisión (naranja) en el Boletín de la Sociedad Geológica Mexicana durante los últimos años, hasta Febrero de 2009. Se indica el promedio de tiempo transcurrido entre el sometimiento de los artículos y su dictamen de aceptación o rechazo (sin datos para 2004). 
Tabla 5. Extracto del padrón de revisores de artículos para el bienio 2007-2008 en el Boletín de la Sociedad Geológica Mexicana.

\begin{tabular}{lcc}
\hline & Revisiones & $\%$ \\
\hline Instituciones mexicanas & 85 & 48.6 \\
UNAM & 47 & 26.9 \\
No UNAM & 38 & 21.7 \\
Miembros del SNI & 71 & \\
Instituciones extranjeras & 90 & 51.4 \\
España & 29 & 16.6 \\
E.U.A. & 22 & 12.6 \\
Alemania & 6 & 3.4 \\
Chile & 4 & 2.3 \\
Suiza & 4 & 2.3 \\
Argentina & 3 & 1.7 \\
Brasil & 3 & 1.7 \\
Italia & 3 & 1.7 \\
Reino Unido & 3 & 1.7 \\
Venezuela & 3 & 1.7 \\
Canadá & 2 & 1.1 \\
Francia & 2 & 1.1 \\
Austria & 1 & 0.6 \\
Colombia & 1 & 0.6 \\
Cuba & 1 & 0.6 \\
Holanda & 1 & 0.6 \\
Japón & 1 & 0.6 \\
Tailandia & & 0.6 \\
\hline
\end{tabular}

\section{Bienio 2005-2006}

México - UNAM México - no UNAM

Francia

E.U.A.

Reino Unido

Japón

Bienio 2007-2008

México - UNAM México - no UNAM

España

Cuba

Rusia

Colombia

India

E.U.A.

Holanda

Italia

Alemania

Argentina

Canadá

Canadá

Austria

Francia

Nicaragua

Perú

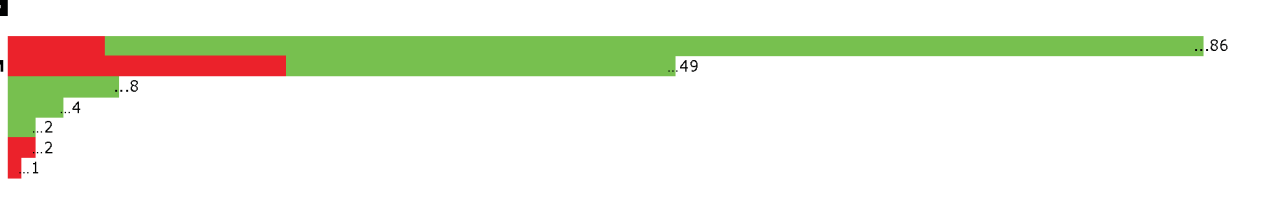

Bienio 2009-2010

México - UNAM

MeU.A.

Rusia
Cuba

Cuba

España
India

Irán

Reino Unido

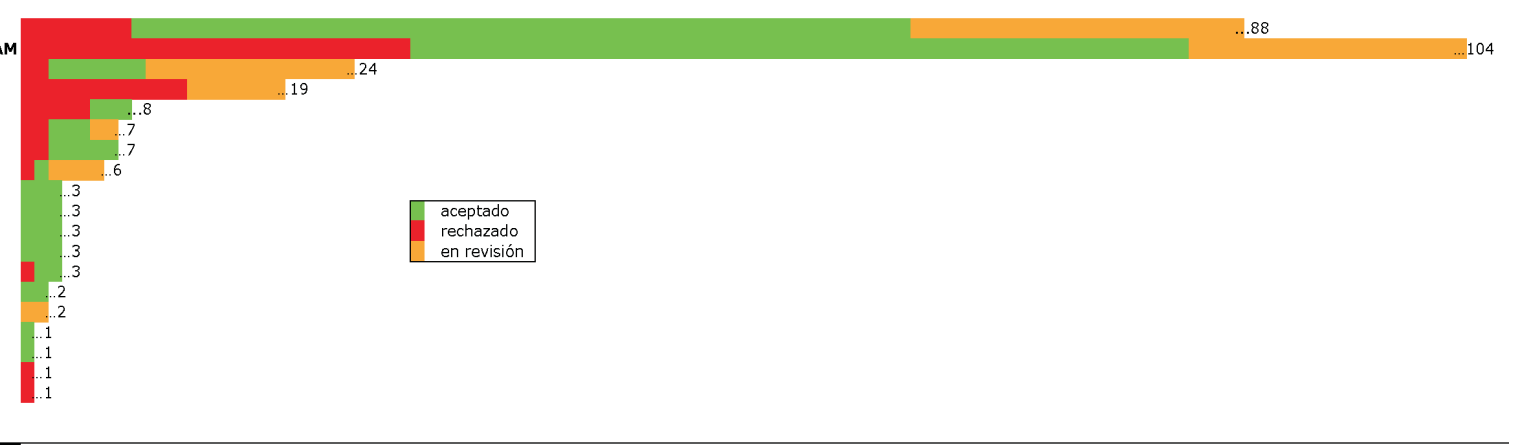

Figura 3. Autores que sometieron trabajos al Boletín de la Sociedad Geológica Mexicana, para los bienios 2005-2006 (152 autores en total, 17 del extranjero), 2007-2008 (296 autores en total, 104 del extranjero) y 20092010 (provisionalmente, 35 autores en total, 9 del extranjero), por países y distinguiendo su entidad de adscripción para México (pertenecientes a la UNAM o no), con indicación del dictamen del artículo o si se encuenta en proceso de revisión. Elaborado en Febrero de 2009. 
Scopus, el servicio bibliográfico en línea de Elsevier B.V. Asimismo, a inicios de 2008 se solicitó el ingreso del Boletín en la Red de Revistas Científicas de América Latina y el Caribe, España y Portugal (Redalyc), y a inicios de 2009 se solicitó su ingreso en SciELO y en eJournals, sin que existan todavía pronunciamientos oficiales al respecto.

\section{Generación de listas de los artículos más descargados}

Las listas de los artículos más descargados, elaboradas para cada trimestre y año, se determinan en base al número de descargas de cada mes, que es ponderado mediante la cantidad promedio de memoria descargada de cada artículo (relativa a la memoria total del mismo) y la cantidad de veces que su URL constituyó la entrada o salida del Boletín. Dichas listas se encuentran en un lugar visible de las páginas del Boletín.

\section{Cambios en la edición técnica}

Hasta mediados de 2008, la edición técnica corría a cargo por entero de la misma persona, quien también lleva a cabo la edición de los artículos de la Revista Mexicana de Ciencias Geológicas. Sin embargo, debido al crecimiento tanto de la Revista como del Boletín esa labor no puede estar a cargo de una sola persona, por lo que se ha buscado el apoyo alternativo de diversas personas para todas las tareas relacionadas con la edición técnica, aunque la mayoría todavía se encuentran en periodo de adaptación. Asimismo, en el Instituto de Geología de la UNAM se ha abierto recientemente un programa de servicio social destinado al apoyo editorial mediante el cual se efectuarán tareas en la edición técnica del Boletín.

\section{Elaboración de un reglamento de permanencia en el Comité Editorial y de una normativa de ética}

Además de la tarea editorial propiamente dicha, ha sido necesario reformular las relaciones entre los miembros del comité editorial, sus responsabilidades y su permanencia en el comité en base a un nuevo reglamento. De la misma forma, es preciso contar con una normativa ética dirigida a autores, revisores y editores, tipificando en ella qué hechos o actitudes son constitutivos de falta y en qué grado. De ambos conjuntos de disposiciones se elaboraron ya borradores completos, que están siendo examinados actualmente por los editores asociados antes de ser sometidos a votación por el resto de miembros del pleno del comité editorial.

\section{El futuro inmediato del Boletín}

En el momento en que fue redactada la presente editorial se encontraban en proceso de edición los tres números del Boletín que serán publicados durante 2009: el número especial "Geología y ambientes deposicionales marinos", un número regular, y el número especial dedicado al "Primer taller iberoamericano de recursos minerales y de apoyo a la pequeña minería, La Habana 2008". Asimismo, están abiertas cuatro convocatorias para otros tantos números especiales: "Avances y paradigmas de la tectónica y la historia geológica del Noreste de México", "Cambio climático y paleoclimatología", "Aplicaciones de SIG y teledetección en Geociencias" y "XI Congreso Nacional de Paleontología, Juriquilla 2009". Teniendo en cuenta que la recepción de trabajos sometidos espontáneamente al Boletín sigue en aumento, seguirán editándose números regulares.

A pesar del carácter histórico que posee el Boletín para las Ciencias de la Tierra en México y su entorno geológico inmediato, hoy en día sigue siendo una revista en proceso de consolidación dentro del medio académico. Resulta evidente que el interés que ha suscitado recientemente el Boletín en muchos académicos tiene mucho que ver con el hecho de pertenecer éste al Índice de Conacyt. Por ello, durante el ejercicio del actual comité editorial se ha tenido especial cuidado en seguir las indicaciones emitidas en el dictamen de Conacyt. Ello, sin olvidar temas que, como la geología urbana, tienen un impacto social directo o que constituyen disciplinas emergentes, ni tampoco aquellas disciplinas en las Ciencias de la Tierra que han recibido relativamente poca atención en el Boletín.

Finalmente, aunque no es a los miembros del comité editorial a quienes corresponde juzgar los méritos del esfuerzo colectivo que ha supuesto y supone editar el Boletín desde 2005 , los avances en los últimos años son notables. Es cierto que persisten muchos aspectos mejorables en diferentes grados y que queda aún bastante camino por andar, pero no es presunción afirmar que ya se han dado los primeros pasos en la dirección correcta. 